\title{
Joint Source-Channel Coding with Inner Irregular Codes
}

\author{
Ragnar Thobaben \\ ACCESS Linnaeus Center \\ School of Electrical Engineering \\ Royal Institute of Technology (KTH), Stockholm, Sweden \\ ragnar.thobaben@ee.kth.se
}

\author{
Laurent Schmalen, Peter Vary \\ Institute of Communication Systems and \\ Data Processing (IND) \\ RWTH Aachen University, Germany \\ $\{$ schmalen,vary\}@ind.rwth-aachen.de
}

\begin{abstract}
We address the optimization of joint source-channel coding schemes for iterative source-channel decoding of firstorder Markov sources. Compared to the traditional design, we propose two novelties: (1) source encoders, providing code words with a minimum Hamming distance $d_{\min } \geq 2$, realized by linear block codes, and (2) irregular channel encoders which are optimized for both the source characteristics and the conditions on the channel. Inner code rates $R_{C}>1$ may be chosen in order to compensate for the additional source redundancy if required. Design examples for the AWGN channel and an overall code rate $R=0.66$ show that the proposed system is able to establish reliable communication within $0.3 \mathrm{~dB}$ of the capacity limit for an interleaver length of approximatively 200000 bits.
\end{abstract}

\section{INTRODUCTION}

During recent years, several ensembles of channel codes were introduced which are capable of providing reliable communication arbitrarily close to the capacity limit of the communication channel (see e.g. [1] for the binary erasure channel). These codes are iteratively decodable and feature an irregular code structure. However, if applied to practical scenarios with limited block lengths, performance is lost, and often a residual bit error rate remains. If the outcome of a source encoder, employing, e.g., sensitive entropy coding schemes, is subject to these residual errors, a severe distortion of the source signal may be the consequence.

As a remedy, several authors focus on joint source-channel coding and decoding for both arithmetic codes (ACs) and variable-length codes (VLCs). This work includes the design of robust ACs and VLCs (e.g. [2], [3]) as well as the joint and iterative source-channel decoding for ACs and VLCs (e.g. [4][6]). However, although these schemes allow to mitigate the impact of residual errors, they lead to a significantly increased decoding complexity. Furthermore, the efficiency in terms of data compression is reduced in many cases.

An alternative to binary entropy coding may be given by the concept of Turbo source coding (TSC, e.g. [7]). TSC schemes employ highly punctured Turbo-like codes in order to achieve the desired compression. On the decoder side, a priori knowledge in form of the source statistics is then exploited to recover the punctured bits. The implementation of TSC within a JPEG-2000 framework in [8] and promising results for noisy transmissions have motivated the authors in [8] to propagate a

This work was supported in part by VINNOVA. new source-channel coding paradigm: source-channel coding should be implemented by a single linear code. The benefits are an increased performance in terms of robustness and efficiency and a significantly reduced complexity.

In this paper, we provide a further example, justifying the concept from [8]. It may be interpreted as a generalization of TSC to non-binary sources with a focus on robustness instead of compression. We address the system optimization for iterative source-channel decoding for non-binary sources if source redundancy due to residual source correlation is exploited for error protection. Residual source correlation can be observed in several practical speech, audio, and image coding schemes. Specific examples from the related literature are summarized in the introduction of [5].

The proposed system design features two important innovations, compared to traditional approaches in this area. We propose (1) to replace the entropy coding of the quantization indices at the source encoder by a linear block code [9], [10] and (2) a subsequent inner irregular channel encoding of the interleaved source encoded data. The additional redundancy from the source coding stage may be compensated by choosing highly punctured inner codes with code rates $R_{C}>1$ if desired.

The first aspect ensures linearity of the joint source-channel coding scheme. It allows for good convergence properties of the iterative decoder and leads to a reduced decoding complexity compared to non-linear approaches like VLCs or ACs [10]. The irregular design of the inner channel encoder allows furthermore for an appropriate adjustment of the channel encoder to both the characteristics of the source encoder and the conditions on the channel. The proposed approach stands thus in contrast to related work [11] where an irregular source code design is employed in order to adapt the source encoder to the channel encoder and the channel conditions.

From a cross-layer design point of view, the coding scheme presented here is beneficial since it leads to an increased flexibility compared to [11]. In practical scenarios where multimedia contents are recorded, saved, and transmitted several times, the proposed scheme requires only to adapt the channel encoder while the source-encoded data remain unchanged. This is in contrast to [11] where for each "transmission" (e.g., storage on a medium or a wireless transmission) the source encoder (i.e., the application) has to re-optimize its parameters 
in order to re-encode the source data.

The remainder of this paper is organized as follows. Section II summarizes the concept of ISCD and introduces the proposed transmitter structure. The optimization of the employed components, based on an EXIT chart analysis, is discussed in Section III. A comparison between the proposed coding scheme and the optimized schemes from [12] for the AWGN channel is finally shown in Section IV.

\section{Proposed Transmission System}

We consider the transmission system depicted in Fig. 1. It describes a source-channel coded transmission of a length- $K$ source vector $\boldsymbol{U}$ over a noisy transmission channel. The source and the channel encoder are separated by an interleaver, and an iterative source-channel decoder is employed.

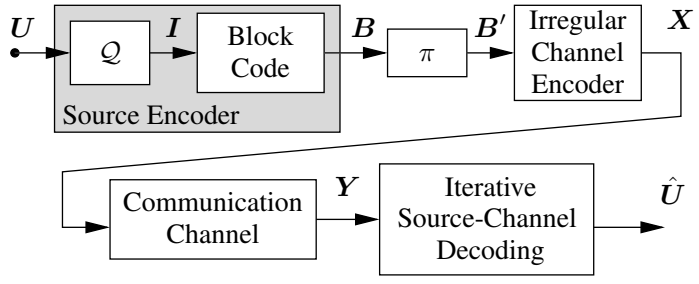

Fig. 1. Model of the transmission system, containing source encoding, channel encoding, and iterative source-channel decoding.

\section{A. Source Model and Joint Source-Channel Encoding}

In order to account for the residual source redundancy, we consider the case where the elements $U_{k}$ of the symbol vector $\boldsymbol{U}$ are non-uniformly distributed and correlated in time. We assume that, after $W$-bit quantization, the residual source correlation in the (quantization) index vector $\boldsymbol{I}$ with elements $I_{k} \in \mathcal{I}$ can sufficiently be described by a Markov process of appropriate order $\mu$. In the following, we restrict ourselves to the standard scenario with $\mu=1$ where the indices $I_{k}$ are characterized by the index-transition probabilities $\operatorname{Pr}\left(I_{k} \mid I_{k-1}\right)$ and their distribution $\operatorname{Pr}\left(I_{k}\right)$.

After quantization, the indices $I_{k}$ are mapped symbol-wise to a binary representation, resulting in a length- $N$ bit vector $\boldsymbol{B}$ with elements $B_{n} \in\{0,1\}$. Therefore, we map the index realizations $i \in \mathcal{I}$ one-to-one to the code words $\boldsymbol{c}_{i}$ of a linear block code with the code word set $\mathcal{C}=\left\{\boldsymbol{c}_{1}, \ldots, \boldsymbol{c}_{|\mathcal{I}|}\right\}$, with $|\mathcal{I}|=2^{W}$, the codeword length $L$, and the code rate $R_{B C}=W / L$. Correspondingly, for a realization $i$ or the index vector $\boldsymbol{I}$ with elements $i_{k}$ the realization $\boldsymbol{b}$ at the output of the source encoder can be expressed as $\boldsymbol{b}=\left[\boldsymbol{c}_{i_{1}}, \ldots, \boldsymbol{c}_{i_{K}}\right]$. Compared to a perfect entropy encoder the mapping to a block code introduces additional redundancy. While this marks a drawback from compression point of view, we obtain a minimum Hamming distance $d_{\min }=2$ between all source realizations $\boldsymbol{b}$. As we will see in Section III, good convergence properties can be expected for the iterative decoder.

A permuted version $\boldsymbol{B}^{\prime}$ of the source bit vector $\boldsymbol{B}$ forms after interleaving $(\pi)$ the input to the irregular channel encoder with rate $R_{C} \geq 1$. A realization of the irregular encoder is shown in Figure 2. The interleaved bit vector $\boldsymbol{B}^{\prime}$ is segmented into a number of $F$ sub-blocks $\boldsymbol{B}_{f}$, with $N_{f}$ denoting the length of the $f$-th block, such that $\boldsymbol{B}^{\prime}=\left[\boldsymbol{B}_{1}, \ldots, \boldsymbol{B}_{F}\right]$. Each sub-block $\boldsymbol{B}_{f}$ is individually encoded by a rate- $R_{f}$ channel encoder $\mathrm{ENC}_{f}$. After channel encoding the resulting code words $\boldsymbol{X}_{f}$ are multiplexed and form the code word $\boldsymbol{X}$ at the output of the irregular encoder. The irregular encoder is specified by the set $\mathcal{C}=\left\{\mathrm{ENC}_{1}, \ldots, \mathrm{ENC}_{F}\right\}$ of employed component encoders and the fractions

$$
\alpha_{f}=N_{f} / N, \quad \text { with } \sum_{f=1}^{F} \alpha_{f}=1,
$$

giving the relative number of bits which are encoded by the respective encoder $\mathrm{ENC}_{f}$.

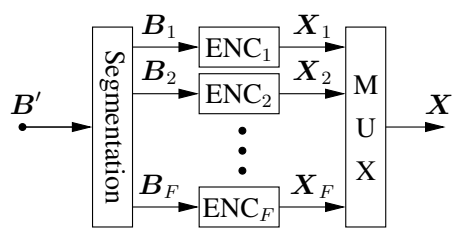

Fig. 2. Irregular inner channel encoder.

\section{B. Transmission and Iterative Source-Channel Decoding}

The transmission of the code vector $\boldsymbol{X}$ is carried out over a noisy memoryless channel, which is characterized by the stationary probability density function (PDF) $\mathrm{p}_{Y \mid X}\left(y_{m} \mid x_{m}\right)$. At the receiver, an iterative source-channel decoder (see e.g. [6], [12]) is employed to recover the source data from the noisy observations in $\boldsymbol{Y}$. It is constituted by two a posteriori probability (APP) decoders for the source and the channel encoder, exchanging extrinsic log-likelihood ratios (LLRs) on the source bits in $\boldsymbol{B}$. After decoding, the vectors $\hat{\boldsymbol{I}}$ and $\hat{\boldsymbol{U}}$ of estimates for the elements of the source vector $\boldsymbol{U}$ and the index vector $\boldsymbol{I}$ are provided. The PDF $\mathrm{p}_{Y \mid X}\left(y_{m} \mid x_{m}\right)$ and the source statistics $\operatorname{Pr}\left(I_{k} \mid I_{k-1}\right)$ and $\operatorname{Pr}\left(I_{k}\right)$ are exploited as $a$ priori information by the iterative decoder.

\section{Overall Redundancy and Code Rate}

According to the different sources of redundancy, the overall code rate $R$ of the coding system can be expressed as

$$
R=\underbrace{\frac{\mathrm{H}\left(I_{k}\right)+(K-1) \cdot \mathrm{H}\left(I_{k} \mid I_{k-1}\right)}{K \cdot \mathrm{H}\left(I_{k}\right)}}_{=: R_{\text {Markov }}} \cdot \underbrace{\frac{\mathrm{H}\left(I_{k}\right)}{L}}_{=: R_{S}} \cdot R_{C} .
$$

It takes into account the contribution $R_{\text {Markov }}$ due to the Markov property of the quantization indices and the contributions $R_{S}$ and $R_{C}$ due to the employed source-channel encoding. Note that the rate $R_{S}$ includes both the redundancy due to the non-uniform distribution of the quantization indices and the redundancy of the employed block code.

To ensure a high flexibility of the proposed source-channel coding scheme for a variety of changing channel conditions, the source redundancy expressed by the product $R_{\text {Markov }} \cdot R_{S}$ has to be chosen sufficiently low. This can be achieved, e.g., by choosing an appropriate block code and can be compensated by a high-rate channel code with $R_{C}>1$ if necessary. The connection to Turbo source coding becomes obvious. 


\section{System OptimizATION}

Due to the interleaved serial concatenation of the source encoder and the channel encoder, the joint optimization of the employed components underlies the same restrictions as the design of serially concatenated channel codes (SCCCs). Therefore, we resume guidelines for the design of SCCCs and discuss their implications on the transmitter optimization for iterative source channel decoding.

\section{A. EXIT Chart Method}

The design of concatenated codes was significantly simplified by the invention of the EXIT chart method (EXtrinsic Information Transfer characteristics). EXIT functions $\mathcal{X}($. characterize the component decoders of an iterative decoder by the amount of mutual information $I_{\text {extr }}$ the extrinsic values at the output of the decoders provide on the corresponding symbols at the encoder, given a certain amount of mutual information $\mathrm{I}_{\text {apri }}$ at their a priori input: $\mathrm{I}_{\text {extr }}=\mathcal{X}\left(\mathrm{I}_{\text {apri }}\right)$. By drawing the EXIT function $\mathrm{I}_{\text {extr }}^{(i)}=\mathcal{X}^{(i)}\left(\mathrm{I}_{\text {apri }}^{(i)}\right)$ of the inner encoder/decoder pair into the same diagram with the inverse EXIT function $\mathrm{I}_{a p r i}^{(o)}=\mathcal{X}^{(o)^{-1}}\left(\mathrm{I}_{\text {extr }}^{(o)}\right)$ of the outer encoder/decoder pair $^{1}$, a prediction of the convergence behavior of the iterative decoder can be obtained by drawing decoding trajectories between the EXIT functions.

To maximize the information exchanged by the component decoders during the iterations, the EXIT functions must fulfill $\mathcal{X}^{(i)}(\mathrm{I})>\mathcal{X}^{(o)^{-1}}(\mathrm{I})$, for all $\mathrm{I} \in[0,1)$; i.e., intersections at $\left(\mathrm{I}_{\text {apri }}, \mathrm{I}_{\text {extr }}\right)<(1,1) \mathrm{bit} /$ channel use have to be avoided. They mark a fix-point of the iterative decoder and lead to a convergence towards high bit error rates. It follows that both EXIT functions $\mathcal{X}^{(i)}($.$) and \mathcal{X}^{(o)}($.$) must reach \left(\mathrm{I}_{\text {apri }}, \mathrm{I}_{\text {extr }}\right)=$ $(1,1) \mathrm{bit} / \mathrm{channel}$ use. As a consequence, the inner encoder must be recursive [13] and the minimum Hamming distance $d_{\min }^{(o)}$ of the outer encoder must satisfy $d_{\min }^{(o)} \geq 2$.

\section{B. Outer Source Encoder}

In the joint source-channel coding scheme depicted in Fig. 1, the source encoder takes the role of the outer channel encoder. Following the guidelines summarized in the previous section, the source encoder has to be chosen such that a minimum Hamming distance between $d_{\text {min }}^{(o)} \geq 2$ is guaranteed. The proposed system design from Section II ensures this; it is thus well-suited for iterative source-channel decoding.

In general, the shape of the inverse EXIT functions $\mathcal{X}^{(o)^{-1}}$ depends on the source characteristics. The amount of source redundancy, which can be associated with the area under the inverse EXIT function $\mathcal{X}^{(o)^{-1}}$, has hereby the most significant impact. As proven in [14] for the binary erasure channel, the area $\mathcal{A}\left(\mathcal{X}^{(o)^{-1}}\right)$ under the inverse EXIT characteristic of the outer decoder corresponds to its code rate $R^{(o)}$,

$$
\mathcal{A}\left(\mathcal{X}^{(o)^{-1}}\right)=R^{(o)},
$$

which is given for the source-channel coding scheme from Figure 1 as $R^{(o)}=R_{\text {Markov }} \cdot R_{S}$.

\footnotetext{
${ }^{1}$ Note that the extrinsic output of the one decoder becomes the a priori input of the other decoder; i.e., we have $\mathrm{I}_{\text {extr }}^{(i)}=\mathrm{I}_{\text {apri }}^{(o)}$ and $\mathrm{I}_{\text {apri }}^{(i)}=\mathrm{I}_{\text {extr }}^{(o)}$.
}

\section{Inner Irregular Channel Encoder}

The area theorem states for the inner code that the area $\mathcal{A}\left(\mathcal{X}^{(i)}\right)$ under the inner EXIT characteristic $\mathcal{X}^{(i)}$ is lower or equal to the ratio of the mutual information $I_{c}$ between the observations from the communication channel and the transmitted symbols and the inner code rate $R^{(i)}$ :

$$
\mathcal{A}\left(\mathcal{X}^{(i)}\right) \leq \mathrm{I}_{c} / R^{(i)},
$$

with the inner code rate $R^{(i)}=R_{C}$ in the case of the sourcechannel coding scheme from Figure 1 .

Since in (2) equality holds only for $R^{(i)} \geq 1$, the inner code should be chosen accordingly. A capacity loss [14] which may increase the decoding threshold towards higher channel signalto-noise ratios (SNR) can be avoided by this. On the other hand, for $R^{(i)} \geq 1$ and EXIT functions fulfilling $\mathcal{X}^{(i)}(\mathrm{I})>$ $\mathcal{X}^{(o)^{-1}}(\mathrm{I})$, for all $\mathrm{I} \in[0,1)$, the area between the two EXIT functions corresponds to the difference between the mutual information $I_{c}$ provided by the channel and the overall code rate $R=R^{(i)} \cdot R^{(o)}$. For the design of coding schemes which are able to operate reliably and close to the capacity limit of the channel, the design goal is to find component codes with good matching EXIT functions such that $\mathcal{X}^{(i)}(\mathrm{I})>\mathcal{X}^{(o)^{-1}}(\mathrm{I})$ is maintained when $R$ approaches $\mathrm{I}_{c}$ and the difference $\mathrm{I}_{c}-R=$ $\mathcal{A}\left(\mathcal{X}^{(i)}(\mathrm{I})\right)-\mathcal{A}\left(\mathcal{X}^{(o)^{-1}}(\mathrm{I})\right)$ tends to zero. Irregular codes are well suited to achieve this goal.

The optimization of irregular codes based on the EXITchart method was extensively discussed in [15]. A fundamental result is that the EXIT function of an irregular code can be obtained as a linear combination of EXIT functions of the employed basis codes. While in the literature only outer irregular codes are addressed, the adoption to the case of inner irregular codes is straight forward. The EXIT function $\mathcal{X}^{(i)}(\mathrm{I})$ can hence be obtained from the EXIT functions $\mathcal{X}^{\left(\mathrm{ENC}_{f}\right)}(\mathrm{I})$ associated with the encoders ENC $_{f}$ according to

$$
\mathcal{X}^{(i)}(\mathrm{I})=\alpha_{1} \cdot \mathcal{X}^{\left(\mathrm{ENC}_{1}\right)}(\mathrm{I})+\ldots+\alpha_{F} \cdot \mathcal{X}^{\left(\mathrm{ENC}_{F}\right)}(\mathrm{I}),
$$

where the coefficients $\alpha_{f}$ are defined in (1). Given the set of basis codes $\left\{\mathrm{ENC}_{1}, \ldots, \mathrm{ENC}_{F}\right\}$, the code optimization aims at the optimization of the coefficients $\alpha_{f}$ such that $\mathcal{X}^{(i)}(\mathrm{I})>\mathcal{X}^{(o)^{-1}}(\mathrm{I})$. In [15], several optimization criteria were investigated such as the minimization of the squared error or the minimization of the absolute error between the EXIT functions $\mathcal{X}^{(i)}(\mathbf{I})$ and $\mathcal{X}^{(o)^{-1}}(\mathbf{I})$ with respect to the constraints

$$
\sum_{f=1}^{F} \alpha_{f}=1 \quad \text { and } \quad \sum_{f=1}^{F} \alpha_{f} R_{f}=R_{C} .
$$

In the design example presented in the next section, we apply the minimization of the squared error as an optimization criterion for the coefficients $\alpha_{f}$.

\section{EXAMPLE}

In order to prove the good performance of the joint sourcechannel coding scheme introduced in Section II, an example for a system optimization following the guidelines from the previous section is given in Section IV-A. The corresponding 
EXIT functions are shown in Fig. 3. For the optimized parameters of the source-channel encoder, simulations for the binary-input AWGN channel are carried out. The results for symbol error rate $S E R=\operatorname{Pr}\left(I_{k} \neq \hat{I}_{k}\right)$ and the distortion $D=\mathrm{E}\left\{\left(U_{k}-\hat{U}_{k}\right)^{2}\right\}$ after decoding, represented by the reconstruction SNR (R-SNR), are given in Fig. 4.

Three reference systems are considered which employ either an optimized mapping with $R_{B C}=1$ for the bit assignment for the quantization indices [16] or a VLC which guarantees a minimum distance $d_{\min }=2$ by accepting only code words with even Hamming weight (even-weight VLC (EW-VLC), see [12]). Channel coding for the optimized mapping is performed with the memory- 5 rate- 1 recursive convolutional code $(75,1)_{8}$. In the EW-VLC case, both a conventional design, employing the memory-3 rate-1 RP-RSC code $(13,3)_{8}$ with $p_{\text {sys }}=0.92$, and an irregular design are applied as described in the following. All considered coding schemes are optimized for an overall code rate $R=0.66$. The corresponding capacity limit lies at $E_{b} / N_{0}^{(c a p)}=1.02 \mathrm{~dB}$.

\section{A. EXIT Chart Analysis}

In the following, the source signal $U$ is realized by a Gaussian autoregressive random process with a correlation coefficient $a=0.9$. It is well suited for modeling the residual source correlation which can be measured for several parameters in practical multimedia coding systems. After 4bit quantization with a scalar Lloyd-Max quantizer, the index sequence $\boldsymbol{I}$ can be sufficiently good approximated by a firstorder Markov process, and the code rate associated with the residual source correlation is given by $R_{\text {Markov }}=0.69$. A block length of $K=50000$ source symbols is considered.

Here, we demonstrate a code design for a target rate $R=$ 0.66 . In order to satisfy $R_{\text {Markov }} \cdot R_{S} \leq R$ (see Section II), we employ a single-parity-check code (SPCC) with rate $R_{B C}=$ $4 / 5$ for source encoding, leading with $R_{S}=0.76$ to an overall source code rate $R_{\text {Markov }} \cdot R_{S}=0.52$. We refer to this coding scheme as parity-check mapping (PC Mapping) in the following. The inverse EXIT function $\mathcal{X}^{(o)^{-1}}$ describing the behavior of the outer SISO source decoder is shown in Figure 3.

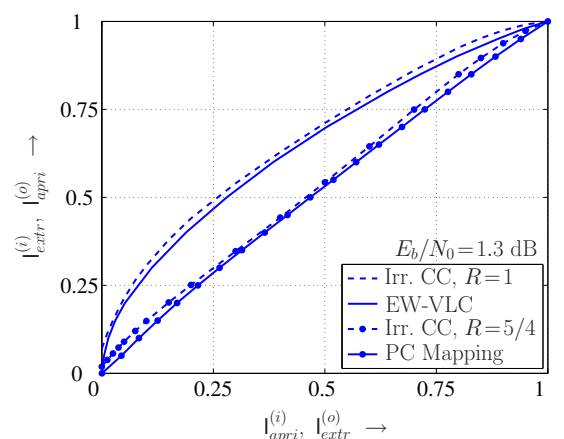

Fig. 3. EXIT chart analysis for two source-channel coding schemes with inner irregular channel codes and an EW-VLC or a SPCC source encoding.

The desired target rate $R$ is now achieved by setting the rate $R_{C}$ of the inner channel code to $R_{C}=5 / 4$. This example demonstrates how a high amount of source redundancy can indeed be compensated after source encoding by an appropriate chosen high-rate channel code with $R_{C}>1$. The connection to Turbo source coding becomes again obvious.

The optimization of the inner irregular channel code (Irr. CC) for the given source parameters and the PC mapping is performed in two steps. In a first step, an appropriate set $\mathcal{C}^{(M C)}=\left\{\mathrm{ENC}_{1}, \ldots, \mathrm{ENC}_{F}\right\}$ of basis codes is selected. Therefore, we choose $F=3$ different randomly punctured recursive systematic convolutional (RP-RSC) codes

$$
\mathcal{C}^{(M C)}=\left\{(11,5)_{8},(13,3)_{8},(17,11)_{8}\right\},
$$

given by the octal representation of the generator polynomials. All codes exhibit a code rate $R_{C}=5 / 4$. The constraints from (3) are thus simplified. The puncturing rates $p_{s}$ and $p_{c}$ of the respective codes, defining the fractions of punctured systematic and punctured code bits, are given in Table I.

\begin{tabular}{|l|ccc|}
\hline & $(11,5)_{8}$ & $(13,3)_{8}$ & $(17,11)_{8}$ \\
\hline$p_{s}$ & 0.99 & 0.93 & 0.93 \\
$p_{c}$ & 0.21 & 0.27 & 0.27 \\
\hline
\end{tabular}

TABLE I

PUNCTURING RATES $p_{S}$ AND $p_{c}$ FOR THE EMPLOYED RP-RSC CODES

In a second step, the coefficients $\alpha_{f}$ are optimized. Therefore, we focus on a signal-to-noise ratio (SNR) $E_{b} / N_{0}=$ $1.3 \mathrm{~dB}$ on the channel and apply the minimization of the squared error between the EXIT functions $\mathcal{X}^{(i)}(I)$ and $\mathcal{X}^{(o)^{-1}}(\mathrm{I})$. For the set $\mathcal{C}^{(M C)}$ from above, we obtain the coefficients

$$
\alpha_{1}=0.4293, \quad \alpha_{2}=0.5265, \quad \text { and } \quad \alpha_{3}=0.0442 .
$$

The EXIT function $\mathcal{X}^{(i)}$ for the resulting inner irregular channel code is included in Figure 3. It closely matches the inverse EXIT function $\mathcal{X}^{(o)^{-1}}$ of the source decoder.

In addition to the EXIT functions for the proposed joint source-channel coding scheme, the EXIT function for an alternative system design, employing the EW-VLC from [12] and a rate- 1 inner irregular channel code, are included in Figure 3 as well. It is similarly well adapted to the source encoder. Accordingly, both systems can be expected to show approximately the same performance. However, the proposed design, employing the PC mapping will turn out to be beneficial due to a reduced complexity.

\section{B. Simulation Results}

The results in Figure 4 show the typical characteristics for reasonably designed source-channel coding schemes under iterative decoding: a region where the iterative decoder fails at low values of the channel SNR $E_{b} / N_{0}$, characterized by high error rates and high distortion, is followed by a narrow transition to a region, where a quasi-error free transmission is obtained. Similar to the bit error rate curves of Turbo or LDPC codes, the transition is characterized by a typical Turbo cliff for the SER which corresponds to a steep decrease in distortion (or increase in R-SNR). 
(a)

(b)

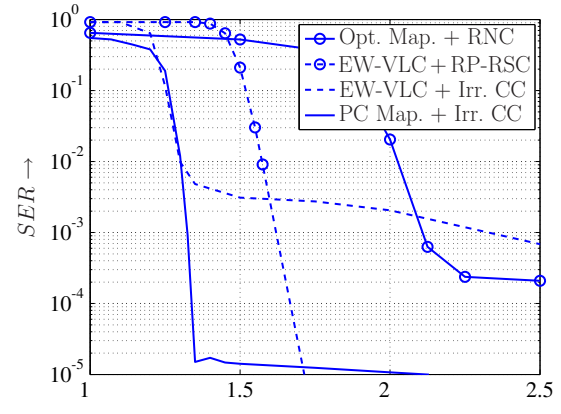

$E_{b} / N_{0}$ in $\mathrm{dB} \rightarrow$

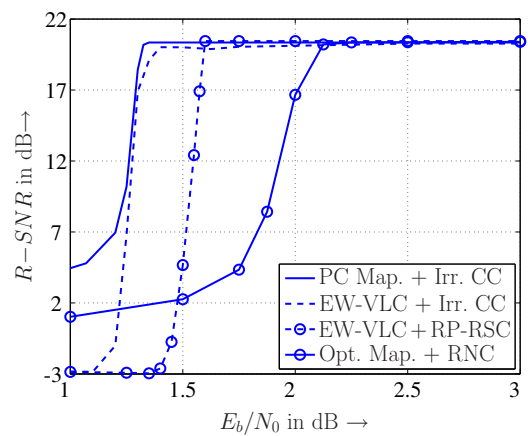

Fig. 4. Results for the binary-input AWGN channel: symbol error rate $(S E R)$ and reconstruction SNR $(R-S N R)$ over the channel SNR $E_{b} / N_{0}$.

For the proposed system, employing the PC mapping and an irregular channel code design, the Turbo cliff can be observed for $1.2 \leq E_{b} / N_{0} \leq 1.35 \mathrm{~dB}$. It is followed by a slightly decreasing residual symbol error rate of about $10^{-5}$. As Figure 4(b) shows, the residual error rate is negligible w.r.t. the distortion such that the clear-channel quality, characterized by the quantization noise, is obtained for $E_{b} / N_{0} \geq 1.32 \mathrm{~dB}$. A quasi error-free transmission is thus achieved $0.3 \mathrm{~dB}$ above the capacity limit of the binary-input AWGN channel.

A comparison to the three reference systems proves the superiority of the proposed scheme: gains of $0.3 \mathrm{~dB}$ and $0.8 \mathrm{~dB}$ in $E_{b} / N_{0}$ can be observed w.r.t. the schemes employing regular channel coding. Slight improvements are obtained over the irregular scheme, employing the EW-VLC from [12] for source coding. This scheme suffers from a high residual symbol error rate, which is caused from insertions or deletions of single symbols due to single bit errors.

Although, the improvements over the EW-VLC-based approach with irregular channel encoding are small, the significance of the proposed method becomes obvious when the complexity of the source decoder is taken into account. Due to the fact that in VLC decoding each bit position has to be considered as a potential starting point of a new VLC word, the employed bit-based SISO VLC decoder [5] leads to an increased complexity. Compared to the proposed scheme, it is increased by a factor which is approximately given by the average block length $\bar{l}$ of the employed VLC. Especially for large symbol alphabets $\mathcal{I}$, this becomes a limiting factor.

\section{CONCLUSION}

We have presented a new transmitter concept for iterativelydecoded source-channel coding schemes. The proposed design features two main novelties: (1) a linear block code is employed for mapping the quantized source symbols to a binary representation, and (2) an irregular code design is applied in order to adjust the channel encoder to the source characteristics and the conditions on the channel. Similar to Turbo source coding schemes, the additional source redundancy may be compensated by puncturing the output of the inner channel encoder to high code rates $R_{C}>1$. As the simulation results for the binary-input AWGN channel show, reliable communication within $0.3 \mathrm{~dB}$ of the capacity limit for a the overall code rate $R=0.66$ can be achieved. The proposed method is superior to approaches, employing variable-length codes and irregular channel encoding, while leading to significant reduction of complexity at the same time.

\section{REFERENCES}

[1] M. G. Luby, M. Mitzenmacher, M. A. Shokrollahi, and D. A. Spielman, "Efficient erasure correcting codes," IEEE Trans. Inf. Theory, vol. 47, no. 2, pp. 569-584, Feb. 2001.

[2] M. Grangetto, E. Magli, and G. Olmo, "Robust video transmission over error-prone channels via error correcting arithmetic codes," IEEE Communications Letters, vol. 7, no. 12, pp. 596- 598, Dec. 2003.

[3] V. Buttigieg, Variable-Length Error-Correcting Codes, Ph.D. thesis, Department of Electrical Engineering, University of Manchester, Manchester, England, 1995.

[4] T. Guionnet and C. Guillemot, "Soft and joint source-channel decoding of quasi-arithmetic codes," EURASIP Journal on Applied Signal Processing, vol. 2004, no. 3, pp. 393-411, Mar. 2004.

[5] R. Thobaben and J. Kliewer, "Low complexity iterative joint sourcechannel decoding for variable-length encoded Markov sources," IEEE Trans. Commun., vol. 53, no. 12, pp. 2054-2064, Dec. 2005.

[6] A. Guyader, E. Fabre, C. Guillemot, and M. Robert, "Joint sourcechannel turbo decoding of entropy-coded sources," IEEE Journal on Sel. Areas in Comm., vol. 19, no. 9, pp. 1680-1696, Sept. 2001.

[7] J. Garcia-Frias and Ying Zhao, "Compression of binary memoryless sources using punctured Turbo codes," IEEE Communications Letters, vol. 6, no. 9, pp. 394-396, Sept. 2002.

[8] M. Fresia and G. Caire, "A practical approach to lossy joint sourcechannel coding," submitted to IEEE Trans. On Information Theory, 2007.

[9] T. Clevorn, P. Vary, and M. Adrat, "Iterative source-channel decoding using short block codes," in Proc. IEEE Int. Conf. Acoust., Speech, Signal Processing, Toulouse, France, May 2006, vol. IV, pp. 221-224.

[10] R. Thobaben, "A new transmitter concept for iteratively-decoded sourcechannel coding schemes," in Proc. IEEE Signal Processing Workshop on Signal Processing Advances in Wireless Communications (SPAWC), Helsinki, Finland, June 2007.

[11] L. Schmalen, P. Vary, T. Clevorn, and B. Schotsch, "Efficient iterative source-channel decoding using irregular index assignments," in Proc. Int. ITG Conf. on Source and Channel Coding, Ulm, Germany, Jan. 2008.

[12] R. Thobaben and J. Kliewer, "Design considerations for iterativelydecoded source-channel coding schemes," in Proc. Allerton Conf. on Communications, Control, and Computing, Monticello, IL, USA, Sept. 2006.

[13] S. ten Brink, "Code characteristic matching for iterative decoding of serially concatenated codes," Annals of Telecommunications, vol. 56, no. 7-8, pp. 394-408, July-August 2001.

[14] A. Ashikhmin, G. Kramer, and S. ten Brink, "Extrinsic information transfer functions: Model and erasure channel properties," IEEE Trans. Inf. Theory, vol. 50, no. 11, pp. 2657-2673, Nov. 2004.

[15] M. Tüchler, "Design of serially concatenated systems depending on the block length," IEEE Trans. Commun., vol. 52, no. 2, pp. 209-218, Feb. 2004.

[16] M. Adrat and P. Vary, "Iterative source-channel decoding: Improved system design using EXIT charts," EURASIP Journal on Applied Signal Processing, vol. 2005, no. 6, pp. 928-941, 2005. 